\title{
Raspberry Pi based Smart Home for Deployment in the Smart Grid
}

\author{
Davinder Pal Sharma \\ Lecturer \\ Department of Physics \\ University of the West Indies \\ Trinidad \& Tobago
}

\author{
Avatar Baldeo \\ Student \\ Department of Physics \\ University of the West Indies \\ Trinidad \& Tobago
}

\author{
Cassiel Phillip \\ Student \\ Department of Physics \\ University of the West Indies \\ Trinidad \& Tobago
}

\begin{abstract}
The Smart Grid is an evolution of the existing electricity grid. It comprises of a two-way communication where electricity and information is exchanged by the consumer and utility to maximize efficiency. Home automation is an important milestone in achieving smart grid and is ever exciting field that has exploded over the past few years. Advancement in technologies have made homes more convenient, efficient and even more secure. Introducing the Raspberry Pi to the world of home automation provides numerous customizations to turn a regular home into a smart home. Raspberry Pi provides a low cost platform for interconnecting electrical/electronic devices and various sensors in a home via the internet network. The main objective of present work is to design a smart home using various sensors which can be controlled and monitored by the Raspberry Pi via the Internet of Things (IoT). This will help the home owners to provide a simple, fast and reliable way to automate their environment. This paper focuses on two aspects of smart home i.e. home security and home automation. Home security system, capable of motion \& disturbance detection at entry points and creating an alarm system with email notification alerts having picture, was implemented to allow real time monitoring for the house. The home automation system was also implemented around the same Raspberry Pi, which includes a smart doorbell, an automated lighting system and a temperature \& humidity controller that turns an air-condition unit or fan on/off automatically under given conditions. Python codes were written for interfacing each sensor and a prototype of smart home was developed. Smart home was fully tested and performance was found satisfactory.
\end{abstract}

\section{General Terms}

Home Automation, Digital System Design, Smart Grid

\section{Keywords}

Raspberry Pi, Smart Home, Home Automation, Home Security, Smart Grid

\section{INTRODUCTION}

The smart grid is an advanced platform to the way we receive electricity today. In earlier times the demand for electricity was substantial compared to that presently. Since the demand for electricity has tremendously increased, a redesign of the current grid system is much needed. With the technology available in these modern times, the smart grid could be designed in such a manner, that it uses digital communications technology to detect and react to local changes in usage. The system will feature a two-way dialog where electricity and information can be exchanged between the consumer and utility. This can increase or decrease the amount of energy a consumer needs by analyzing the feedback of the two-way dialog. The transfer of electricity and information between consumer and utility would increase efficiency, reliability and security. The smart grid also enables renewable energy technology to be integrated into the system for a greener, more environmentally friendly method of obtaining energy, thus reducing a percentage of dependency on fossil fuel [1-3].

Home Automation can be considered as an act of using electronic systems/devices and programming them to replace a number of human interactions for the control of basic home functions. This operates on the base of connecting sensors and devices to the IoT. IoT can be considered as a network of physical objects which can be accessed via the Internet. For objects to considered IoT based, networks need to be converted to an IP-based network for proprietary protocols. The object being connected to the internet can represent itself digitally thereby being controlled from anywhere there is an internet connection. These objects have ability to transmit and receive data over a network without human-tohuman or human-to-computer interaction. This also means that more data can be gathered from these objects, even at a number of places with real-time information being presented from the objects. This super task can increase efficiency, safety and security [4].

The smart home is supposed to be in regular interaction with its internal and external environments. The external environment consists of all the entities belonging to the smart grid and the internal environment consists of all appliances and devices belonging to the smart home, which are centrally managed by an entity in it [5]. A smart home having automated system can be created to establish control of certain aspects of a home. This enhances security and efficiency of a home, which also gives real time monitoring via the internet. Apart from controlling the sensors and components via a device connected to the internet, a centrallycontrolled panel having an LCD screen with keypad or an LCD touchscreen can be used to control many of the applications of home automation [6-7]. Some applications of home automation include automated lights, which can be programed to turn $\mathrm{ON}$ when someone enters a room, or depending on the time of day or turn $\mathrm{ON}$ via a manual command over the internet. Security system, which can be controlled with real time monitoring of the house, where different sensors used in the system can be programed to do particular things such as take photos, turn on a siren, send email alerts and much more. Another application of home automation is a smart thermostat, which can be controlled via 
the internet. The thermostat can control the heating system or air condition system in the house and adjust it to the desired temperature. The thermostat can also be programed to control the system turning it $\mathrm{ON}$ or OFF depending on the time of day or depending on the temperature outside. The total market value for European smart homes market will be worth $\$ 13.81$ Billion by 2020 at an estimated compound annual growth rate (CAGR) of $18.17 \%$ [8].

The Raspberry $\mathrm{Pi}$ and the subject of home automation is a remarkable one. The Raspberry Pi can be used to automate a home at a relatively low cost. It operates on the concept as the IoT. There are numerous things that make the Raspberry Pi essential for home automation but the one that stands out the most has to be the remarkably affordable cost. The vast amount of sensors at extremely low cost makes it's superb for home automation. A security system to be installed into a house can be very costly from security companies, however with some skills and time the Raspberry $\mathrm{Pi}$ can be programed to be a security system with as much sensors as you desire, for a fraction of the cost [9-10]. Figure 1 shows the Raspberry Pi B model with its specifications.

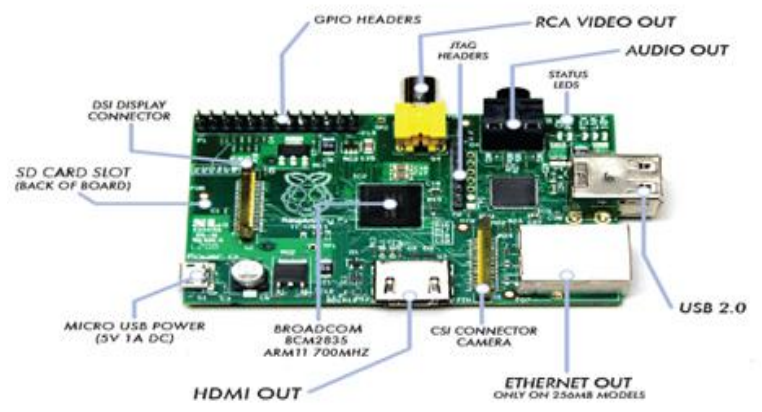

Fig. 1: Raspberry Pi B Model [9]

In the present study an IP-based network was established, Python codes were written for the sensors, which were connected to the $\mathrm{Pi}$, a command was then carried out from the network website, which was processed by the $\mathrm{Pi}$ and reacted with the connected sensors. The purpose of present study is to build a system of interconnected devices and sensors, which allow the user to control and monitor certain electrical/electronic devices in their home via the internet from the Raspberry Pi. This means implementing a system which allows real time monitoring of the home, also forwarding emails to the user when certain devices are triggered.

\section{EXPERIMENTAL DETAILS}

Block diagram of a Raspberry pi based smart home is shown in Figure 2. Main controller unit was built around Raspberry $\mathrm{Pi}$, Piface rack and Piface digital expander. Controller was attached with Wi-Fi module, smart phone, computer, LCD module, power supply and various sensors for home automation and home security features.

The Raspberry Pi operates on a LUNIX based open source operating system called Raspbian OS. This allows more control and flexibility in the software therefore making it easy to program the Pi. The Raspberry Pi communicates with the attached devices and sensors through PYTHON codes to control their functions [11]. The Raspbian operating system was installed onto Raspberry pi, which was obtained by downloading NOOBS onto the SD card from the manufacturer's website [12].

PiFace Rack, which is an expansion board with 4 set of 26 GPIO pins, was used to connect Piface digital expander and Adafruit LCD Pi plate with the Raspberry Pi. Piface digital expander was used to increase number of digital I/Os and LCD Pi plate was used to display the status of Raspberry Pi.

All the sensors for home automation and security applications e.g. PIR infrared motion sensor, magnetic contact switch (Reed switch), siren, speaker, LEDs, push buttons, DHT 11 sensor, DC fan etc. were connected to Raspberry Pi through PiFace digital board.

Main controller was also connected to the Wi-Fi module to obtain the access of internet and html page using smart phone/computer so as to control/access sensors and devices of the home anytime, anywhere.

Table 1 shows the list of major components used during this study. A prototype of smart home was also developed, which is shown in Figure 3 along with controller unit.

Table 1. List of Major Components

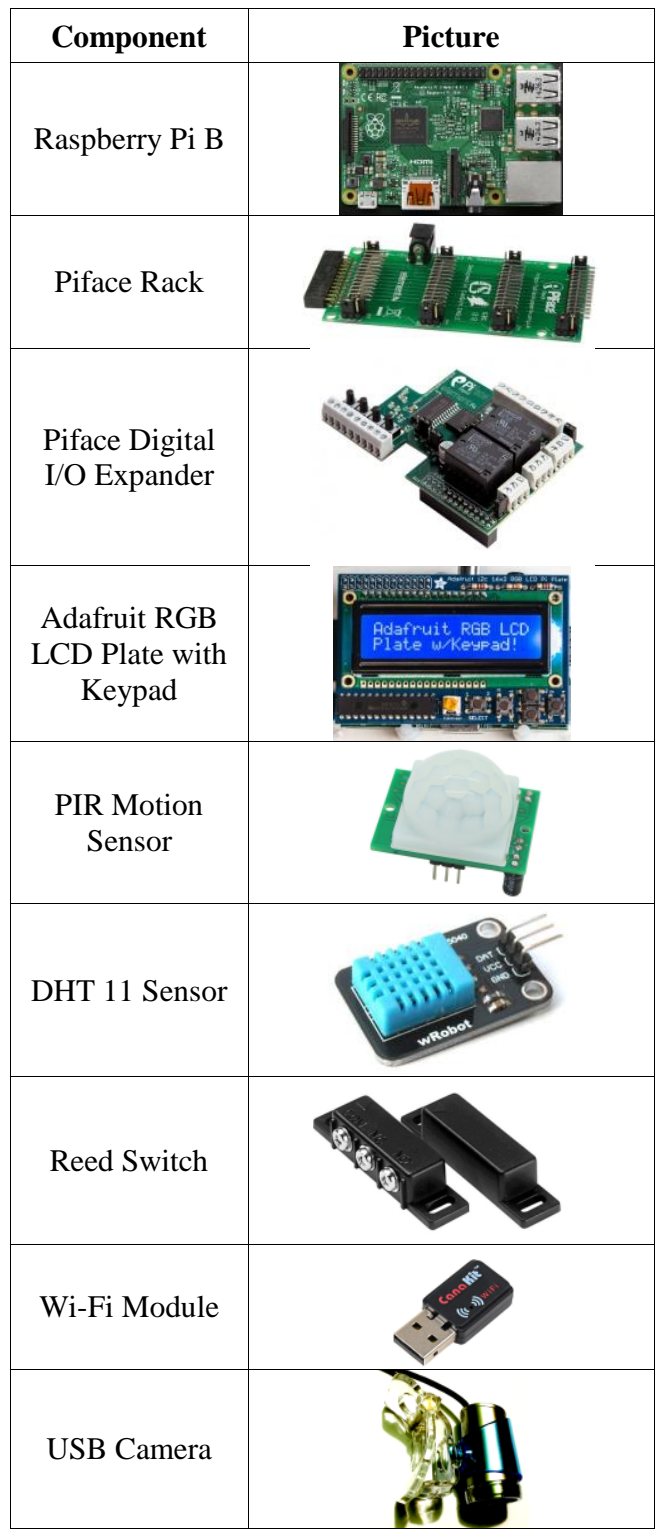




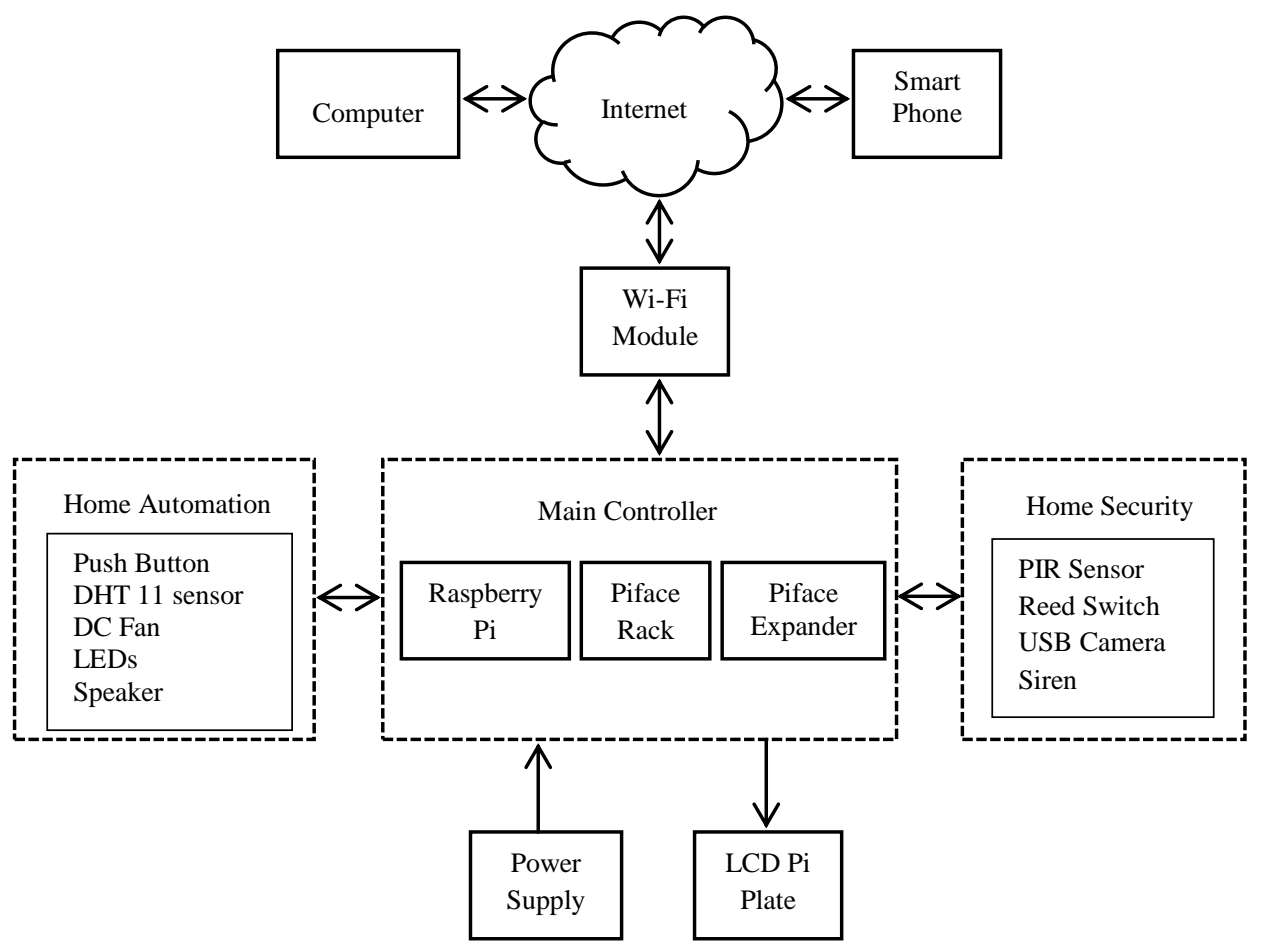

Fig 2: Block diagram of Raspberry Pi based Smart Home

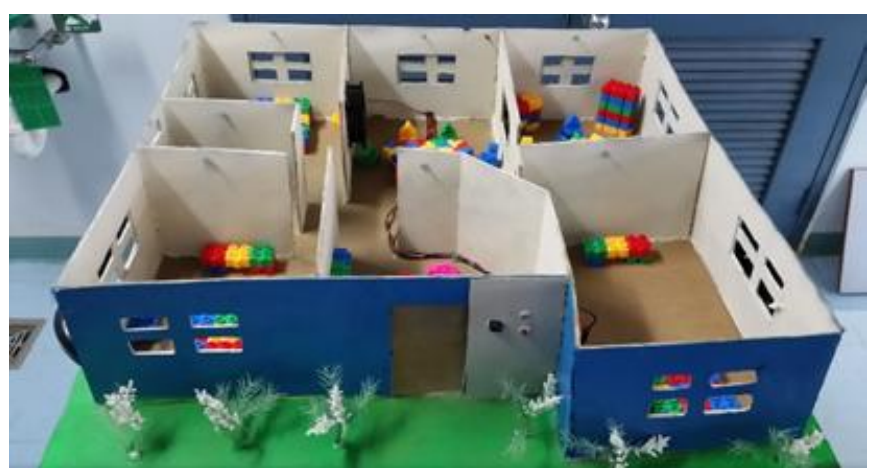

(a)

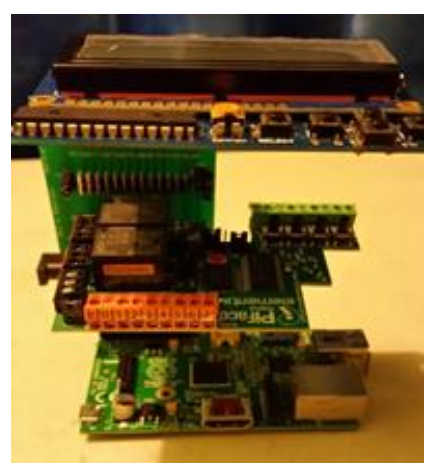

(b)

Fig 3: (a) Prototype of Smart Home (b) Main Controller

\section{RESULTS AND DISCUSSION}

Implementation details and results related to the two aspects of smart home i.e. home security and home automation are discussed here.

\subsection{Home Security}

Home security system, capable of motion \& disturbance detection at entry points along with security alarm system having email notification alerts containing picture, was implemented to allow real time monitoring of the home anywhere and anytime.

The Raspberry Pi based home security system was built using Adafruit RGB positive $16 \times 2$ LCD Pi plate, Wi-Fi module, PIR sensor, USB camera (web cam), Reed switch and internet access point. To activate the security system, keypad of the
LCD Pi plate was used. Security alarm can be armed or disabled by pressing up or left keys of keypads for 3 seconds. Figure 4 indicates the status of alarm on LCD Pi plate. Upon activating the alarm, an audio output is sent to $3.5 \mathrm{~mm}$ audio jack of Raspberry pi and then "System is Armed" sound can be heard via self-powered audio speaker. All the devices mentioned above will also become active.

When an object moves within the range of PIR sensor, a signal is sent to the controller, which initiates the webcam. Webcam snaps a photo which is stored onto memory card of Raspberry Pi. The stored photo is then forwarded via email to the owner with the title "Motion notification" as shown in Figure 5. 

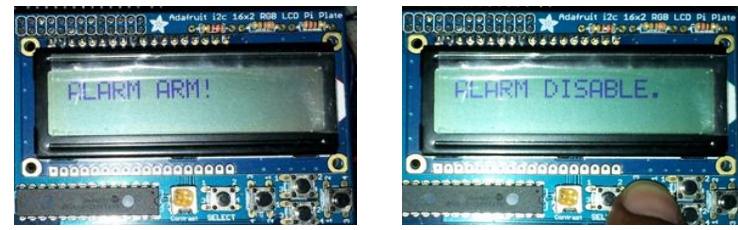

Fig. 4 Home security alarm system status

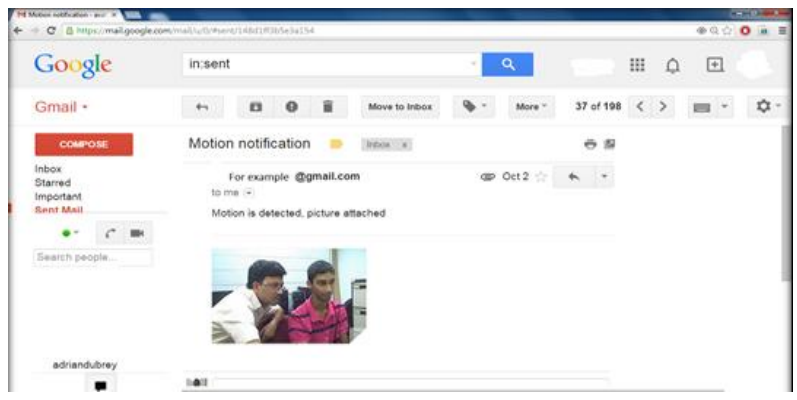

Fig. 5 Email notification upon motion detection

When the door of home equipped with reed switch is opened as shown in Figure 6, the alarm system detects it as a breach, which turns ON the siren and a message "System Breached" is heard on the speaker. At the same time an email is sent to the owner with title "Door Open" and picture around the door. Messages, notification titles, recipient email ID etc. were entered into system through python scripts.

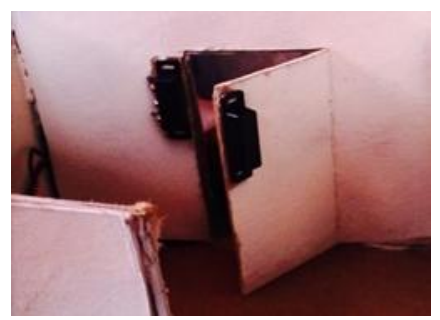

Fig. 6 Entry detection using Reed Switch

\subsection{Home Automation}

The home automation system was also implemented around the same Raspberry Pi, which includes a smart doorbell, an web based automated lighting system and a temperature \& humidity controller that turns an air-condition unit or fan on/off automatically under given conditions.

Figure 7 shows smart door bell, which was built using a push button, LEDs and a speaker. When the push button is pressed, LED 1 will turn ON for 15 seconds and voice message "Someone will attend you shortly" will be heard through speaker. After LED 1 cutoff, LED 2 will be ON for $10 \mathrm{sec}$. and voice message "Sorry, no one is at home, please come again later" will be heard

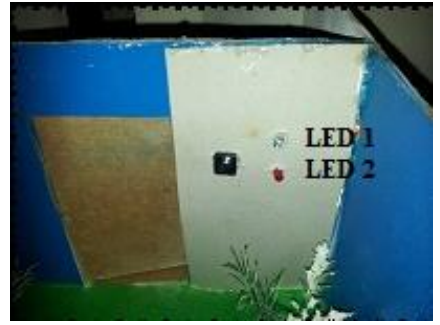

Fig. 7 Smart door bell
Home automated light system was designed using HTML and LEDs. A webpage was developed to control the lights in the home automatically from an internet using a computer, tablet or even a cell phone. This system uses a server address hosted by the Raspberry Pi itself, which is displayed on the LCD Pi plate during start up. Figure 8 shows the web page, which was created to control/check the status of lights and other sensors discussed earlier.

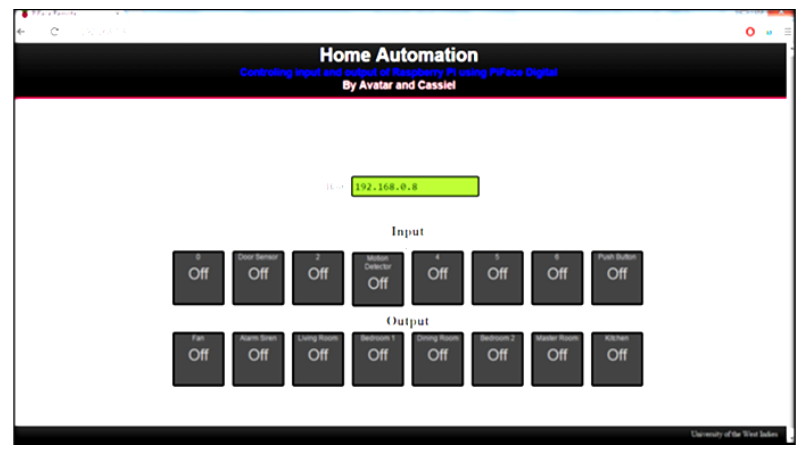

Fig. 8 Webpage to control the smart home

Each button on the webpage is clickable. Buttons on the input section senses signals from the controller. According to which input channel/sensor is in use, the respective button will show $\mathrm{ON}$ and its colour will get changed from grey to sky blue. When any button of the output section is pressed, the respective output would show $\mathrm{ON}$ in red.

To control temperature and humidity inside the home i.e. to control air conditioner (we have used DC fan here), DHT 11 sensor was used. We configured select button of LCD Pi plate, which when pressed displays the temperature and humidity of room/house for 60 seconds as shown in Figure 9 and then returns to its home screen. When the temperature crosses threshold, $28^{\circ} \mathrm{C}$ in our case, the fan/AC will be turned on automatically or also can be turned ON/OFF remotely through the website by pressing Fan button. When the temperature drops down to $28^{\circ} \mathrm{C}$, the fan/AC will cut back off and the process is repeated.

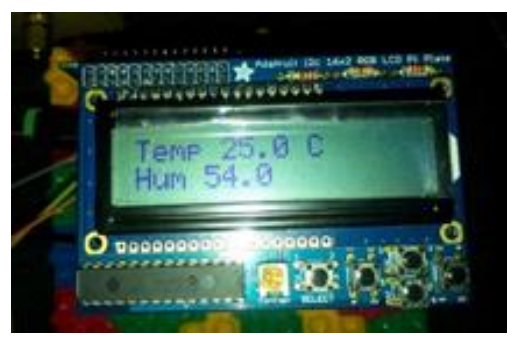

Fig. 9 Temperature/Humidity display

\section{CONCLUSION}

Very soon in near future, the traditional grids of today will evolve into a robust, effective, environment friendly and energy efficient system known as the Smart Grid. Even our home will undergo its own transformation towards the smart homes that will be in constant interaction with the grid in an effort for better energy management and full home automation to ensure comfort, security and privacy.

Present paper sought to design a smart home using various sensors to be controlled and monitored by the Raspberry Pi via the IoT. It is focused on two aspects of smart home i.e. home security and home automation. For home security, the Raspberry Pi is programed to operate as an alarm system in 
which it detects intrusion at entry points along with motion within the home and where email alerts can be sent with pictures to allow real time monitoring of the home. Home automation included a smart door bell, humidity and temperature control via a fan or air-conditioning system of the home. This system is also equipped with automated lights and virtual switches for controlling lights and appliances in the home remotely using external and / or internal networking with the Raspberry Pi via an HTML page.

Full functionality of prototype indicates that devices like Raspberry Pi can play very important role in designing smart home of the future at very low cost. An energy aware smart home can be developed using Raspberry Pi and other sensors.

\section{ACKNOWLEDGMENTS}

Authors are thankful to UWI T\&T RDI Fund to provide necessary financial support to carry out present work through project on "Capacity Building and Research on Smart Grid Technology in the Caribbean Region".

\section{REFERENCES}

[1] X. Fang et. Al. , " Smart grid- The new and improved power grid: A Survey”, IEEE Communication Surveys \& Tutorials, , 2012, Vol. 14, No.4, 944-980.

[2] H. Gharavi and R. Ghafurian, "Smart grid: The electric energy system of the future", Proceedings of the IEEE, 2011, Vol. 99, No. 6, 917 - 921.

[3] A. Ipakchi and F. Albuyeh, "Grid of the future", IEEE Power \& Energy Magazine, 2009, Vol. 7, No. 2, 52-62.
[4] Bassi, Alessandro. Enabling Things to Talk: Designing IoT Solutions with the IoT Architectural Reference Model, 2013, Vol. 1 No. 1, 1-12.

[5] N. Komninos, E. Philippou and A. Pitsillides, "Survey in smart grid and smart home security: issues, challenges and countermeasures", IEEE Communication Surveys \& Tutorials, 2014, Vol. 16, No. 4, 1933-1954.

[6] Dennis, A. K. 2013. Raspberry Pi Home Automation with Arduino. Packt Publishing.

[7] Wei, L. Wei, L. Xin, L. 2013. Design and implement on smart home system. In proceedings of the Fourth International Conference on Intelligent Systems Design and Engineering Applications.

[8] European Smart Homes Market by Products (Security, Access, Lighting, Entertainment, Energy Management Systems, HVAC, and Ballast \& Battery Pack), Services (Installation \& Repair, Renovation \& Customization) \& Country - Global Forecasts and Analysis 2013 - 2020.

[9] Upton, E. Halfacree, G. 2014 Raspberry Pi User Guide, $3^{\text {rd }}$. Ed. Wiley.

[10] Richardson, M. Wallace, S. 2014 Getting Started with Raspberry $\mathrm{Pi}, 2^{\text {nd }}$. Ed. Maker Media Inc.

[11] Bradbury, A. Everard, B. 2014 Learning Python with Raspberry Pi. Wiley.

[12] FOUNDATION, RASPBERRY PI. 2014. NOOBS $\begin{array}{llll}\text { SETUP. } & \text { Accessed } & 11 & 2014 .\end{array}$ http://www.raspberrypi.org/help/noobs-setup/. 